Portland State University

PDXScholar

Electrical and Computer Engineering Faculty

Publications and Presentations

Electrical and Computer Engineering

$10-1-1976$

\title{
Analytic modeling of gain-switched lasers. II. Laser amplifiers
}

Lee W. Casperson

Portland State University

Follow this and additional works at: https://pdxscholar.library.pdx.edu/ece_fac

Part of the Electrical and Computer Engineering Commons

Let us know how access to this document benefits you.

Citation Details

Casperson, L. W. (1976). Analytic modeling of gain-switched lasers. II. Laser amplifiers. Journal of Applied Physics, 47 (10), 4563-4571

This Article is brought to you for free and open access. It has been accepted for inclusion in Electrical and Computer Engineering Faculty Publications and Presentations by an authorized administrator of PDXScholar. Please contact us if we can make this document more accessible: pdxscholar@pdx.edu. 


\title{
Analytic modeling of gain-switched lasers. II. Laser amplifiers
}

\author{
Lee W. Casperson \\ School of Engineering and Applied Science, University of California, Los Angeles, California 90024
} (Received 6 October 1975)

\begin{abstract}
The pulse transfer characteristics of gain-switched laser amplifiers are investigated analytically. New closedform solutions have been obtained including arbitrary space- and time-dependent pump distributions. The general results are specialized to the important cases of uniform and traveling-wave excitation. Input-pulse shapes considered include step functions, practical oscillator output pulses, and also the inherent spontaneous-emission noise. The results lead to improved models for pulse amplifiers and mirrorless traveling-wave lasers. By proper choice of the pump characteristics arbitrary output-pulse waveforms can be synthesized.
\end{abstract}

PACS numbers: $42.60 . \mathrm{N}, 42.60 . \mathrm{L}, 42.60 . \mathrm{C}$

\section{INTRODUCTION}

One of the principal uses of lasers is in light-pulse amplification. The obvious function of such an amplifier is to increase the energy density in a short optical signal; and there are numerous well-known applications of such pulses in the industrial, military, and fusion research areas. Other consequences of pulse amplification which may or may not be desirable, include alteration of the frequency spectrum, temporal shape, and timing of the propagating pulse. Because of the wide applications of pulse amplifiers, it is important that their transfer characteristics be thoroughly understood. The purpose of the present work is to develop new analytical models which explicitly account for space- and timedependent pumping distributions in the laser amplifier. A time dependence of the pumping is inevitable, and in traveling-wave amplifiers a spatial dependence is purposely introduced. Previous analytical models have always assumed that no pumping occurs during the transit of the optical pulse. ${ }^{1-3}$

In a recent work the properties of gain-switched laser oscillators have been considered in detail, and outputpulse characteristics have been derived for several practical laser configurations. ${ }^{4}$ The present study of laser amplifiers is developed from the same basic saturation equations. In Sec. II the equations are solved for the problem of pulse propagation in a saturating amplifier with arbitrary pumping during the pulse transit. The implications of these results are explored in Sec. III where stationary- and traveling-wave pump models are considered together with a step-function input intensity. An important model in practice is an amplifier in which the gain rises uniformly along the amplifier length due to a decaying transfer of excitation from some other state of the system. Initially the gain is small so the leading edge of the pulse grows more slowly than the later portions. Farther along the amplifier the gain has achieved its maximum value by the time the pulse arrives. Thus the leading edge of the pulse ultimately grows and saturates the amplifier at the expense of the pulse tail. The transfer characteristics with traveling-wave excitation are also analyzed and this limit applies to recent Blumlein and electron-beampumped devices such as the $3371-\AA$ nitrogen laser ${ }^{5}$ and the $1600-$ and $1100-\AA$ hydrogen lasers ${ }^{6,7}$
The step-function input is the simplest function to treat analytically, but it is not a natural pulse shape for a gain-switched oscillator output. In Sec. IV the transfer characteristics of prepumped laser oscillators are derived using realistic oscillator pulse shapes which have been obtained previously. ${ }^{4}$ With exponentially rising input pulses stationary solutions are obtained having effective velocities greater than the group velocity. The pulse propagaion formalism is also useful for pulse shaping. By proper choice of the pump distribution an arbitrary output-pulse shape can be achieved, and the details of this procedure are examined in Sec. $V$. In Sec. VI the propagation equations are applied to unsaturated laser amplifiers and the effects of spontaneous relaxation are included.

\section{DERIVATION OF PROPAGATION EQUATIONS}

The first objective of this analysis is to obtain an explicit expression relating the output intensity to the input intensity in a saturating laser amplifier. Various applications of these results are considered in Secs. III and IV. The basic saturation equations for a narrow intensity spectrum $I(z, t)$ in a homogeneously broadened laser amplifier are ${ }^{4}$

$$
\begin{aligned}
& \frac{\partial I(z, t)}{\partial z}+\frac{1}{v_{g}} \frac{\partial I(z, t)}{\partial t}=h \nu_{l} s\left[I(z, t)+I_{0}\right] N(z, t)-\gamma I(z, t), \\
& \frac{\partial N(z, t)}{\partial t}=S(z, t)-s I(z, t) N(z, t)-\frac{N(z, t)}{\tau_{2}},
\end{aligned}
$$

where $N(z, t)$ is the population inversion, $v_{g}$ is the group velocity, $\gamma$ is the distributed loss coefficient, $S(z, t)$ is the pump function, and $\tau_{2}$ is the inversion lifetime. The spontaneous-emission input is approximately $I_{0}$ $=\pi h \nu_{l} \Delta \nu_{h} / 2 A$, where $A$ is the cross-sectional area, and the detector is assumed to intercept a solid angle corresponding to a blackbody mode. Equations (1) and (2) are two coupled equations for the unknown functions $I(z, t)$ and $N(z, t)$. With highly dispersive laser media it is also possible that the group velocity would be a space- and time-dependent function. ${ }^{8}$ For practical high-power lasers, however, the dispersion effects are small compared to the basic saturation phenomena of interest here and $v_{g}$ is regarded as a constant.

In propagation studies it is generally useful to intro- 
duce the new time and space parameters

$$
\tau=t-z / v_{g}, \quad \zeta=z .
$$

In terms of these variables the intensity and inversion functions are governed by the simpler equations

$$
\begin{aligned}
& \frac{\partial I(\zeta, \tau)}{\partial \zeta}=h \nu_{l} s\left[I(\zeta, \tau)+I_{0}\right] N(\zeta, \tau)-\gamma I(\zeta, \tau), \\
& \frac{\partial N(\zeta, \tau)}{\partial \tau}=S(\zeta, \tau)-s N(\zeta, \tau) I(\zeta, \tau)-\frac{N(\zeta, \tau)}{\tau_{2}}
\end{aligned}
$$

Equation (5) is a linear first-order equation in $\tau$ which may be integrated yielding ${ }^{9}$

$$
\begin{aligned}
N(\zeta, \tau)= & \exp \left[-s \int_{-\infty}^{\tau} I\left(\zeta, \tau^{\prime}\right) d \tau^{\prime}-\tau / \tau_{2}\right] \\
& \times \int_{-\infty}^{\tau} S\left(\zeta, \tau^{\prime}\right) \exp \left[s \int_{-\infty}^{\tau^{\prime}} I\left(\zeta, \tau^{\prime \prime}\right) d \tau^{\prime \prime}+\tau^{\prime} / \tau_{2}\right] d \tau^{\prime},
\end{aligned}
$$

where the reasonable initial condition $N(\zeta,-\infty)=0$ has been applied. When $\mathrm{Eq}$. (6) is substituted into Eq. (4) one obtains the final governing equation

$$
\begin{aligned}
& \frac{\partial I(\zeta, \tau)}{\partial \zeta}=h \nu_{l} s\left[I(\zeta, \tau)+I_{0}\right] \exp \left(-s \int_{-\infty}^{\tau} I\left(\zeta, \tau^{\prime}\right) d \tau^{\prime}-\frac{\tau}{\tau_{2}}\right) \\
& \quad \times \int_{-\infty}^{\tau} S\left(\zeta, \tau^{\prime}\right) \exp \left(s \int_{-\infty}^{\tau^{\prime}} I\left(\zeta, \tau^{\prime}\right) d \tau^{\prime \prime}+\frac{\tau^{\prime}}{\tau_{2}}\right) d \tau^{\prime}-\gamma I(\zeta, \tau) .
\end{aligned}
$$

As our first example we consider the evolution of a saturating pulse in a laser amplifier in which there is no spontaneous emission or relaxation $\left(\tau_{2}=\infty, I_{0}=0\right)$ and distributed losses are negligible $(\gamma=0)$. Thus Eq. (7) can be written

$$
\begin{aligned}
\frac{\partial I(\zeta, \tau)}{\partial \zeta}= & h \nu_{l} s I(\zeta, \tau) \exp \left(-s \int_{-\infty}^{\tau} I\left(\zeta, \tau^{\prime}\right) d \tau\right) \\
& \times \int_{-\infty}^{\tau} S\left(\zeta, \tau^{\prime}\right) \exp \left(s \int_{-\infty}^{\tau^{\prime}} I\left(\zeta, \tau^{\prime \prime}\right) d \tau^{\prime \prime}\right) d \tau^{\prime}
\end{aligned}
$$

In the following paragraphs the exact general solution of this equation is derived. Applications and other solutions are considered in Secs. III-VI.

In terms of the new intensity function $C(\zeta, \tau)$ $=s \int_{-\infty}^{\tau} I\left(\zeta, \tau^{\prime}\right) d \tau^{\prime} \mathrm{Eq}$. (8) reduces to

$$
\begin{aligned}
\frac{\partial^{2} C(\zeta, \tau)}{\partial \zeta \partial \tau}= & -h \nu_{l} s \frac{\partial}{\partial \tau} \exp [-C(\zeta, \tau)] \\
& \times \int_{-\infty}^{\tau} S\left(\zeta, \tau^{\prime}\right) \exp \left[C\left(\zeta, \tau^{\prime}\right)\right] d \tau^{\prime} .
\end{aligned}
$$

The order of differentiation can be interchanged on the left-hand side of this equation. Then an integration by parts with respect to $\tau$ yields

$$
\begin{aligned}
\frac{\partial C(\zeta, \tau)}{\partial \zeta}= & -h \nu_{l} s \exp [-C(\zeta, \tau)] \int_{-\infty}^{\tau} S\left(\zeta, \tau^{\prime}\right) \exp \left[C\left(\zeta, \tau^{\prime}\right)\right] d \tau^{\prime} \\
& +h \nu_{l} s \int_{-\infty}^{\tau} S\left(\zeta, \tau^{\prime}\right) d \tau^{\prime}
\end{aligned}
$$

If both sides of $\mathrm{Eq} .(10)$ are multiplied by the new function $D(\zeta, \tau)=\exp [C(\zeta, \tau)]$, one obtains

$$
\begin{aligned}
\frac{\partial D(\zeta, \tau)}{\partial \zeta}= & -h \nu_{l} s \int_{-\infty}^{\tau} S\left(\zeta, \tau^{\prime}\right) D\left(\zeta, \tau^{\prime}\right) d \tau^{\prime}+h \nu_{l} s D(\zeta, \tau) \\
& \times \int_{-\infty}^{\tau} S\left(\zeta, \tau^{\prime}\right) d \tau^{\prime}
\end{aligned}
$$

Differentiation with respect to $\tau$ gives

$$
\frac{\partial^{2} D(\zeta, \tau)}{\partial \tau \partial \zeta}=h \nu_{l} s \int_{-\infty}^{\tau} S\left(\zeta, \tau^{\prime}\right) d \tau^{\prime} \frac{\partial D(\zeta, \tau)}{\partial \tau}
$$

If the order of differentiation on the left-hand side is interchange and the new function $E(\zeta, \tau)=\partial D(\zeta, \tau) / \partial \tau$ is introduced, then $\mathrm{Eq}$ 。(12) can be integrated with respect to $\zeta$ and the result is

$$
E(\zeta, \tau)=E(0, \tau) \exp \int_{0}^{\zeta} h \nu_{l} s \int_{-\infty}^{\tau} S\left(\zeta^{\prime}, \tau^{\prime}\right) d \tau^{\prime} d \zeta^{\prime},
$$

where it is assumed that amplification begins at the coordinate $\zeta=0$. From the previous definitions it follows that $E(0, \tau)$ is given by

$$
E(0, \tau)=\frac{\partial D(0, \tau)}{\partial \tau}=s I(0, \tau) \exp \left(s \int_{-\infty}^{\tau} I\left(0, \tau^{\prime}\right) d \tau^{\prime}\right)
$$

Equation (13) may be integrated directly with respect to $\tau$, and one finds that $D(\zeta, \tau)$ is given by

$$
\begin{aligned}
D(\zeta, \tau)= & s \int_{-\infty}^{\tau} I\left(0, \tau^{\prime}\right) \exp \left[s \int_{-\infty}^{\tau^{\prime}} I\left(0, \tau^{\prime \prime}\right) d \tau^{\prime \prime}\right. \\
& \left.+\int_{0}^{\zeta} h \nu_{l} s \int_{-\infty}^{\tau^{\prime}} S\left(\zeta^{\prime}, \tau^{\prime \prime}\right) d \tau^{\prime \prime} d \zeta^{\prime}\right] d \tau^{\prime}+1,
\end{aligned}
$$

where the condition $D(\zeta,-\infty)=1$ has been employed. Now the intensity is

$$
\begin{aligned}
I(\zeta, \tau)= & \frac{1}{s D(\zeta, \tau)} \frac{\partial D(\zeta, \tau)}{\partial \tau} \\
= & I(0, \tau) \exp \left(s \int_{-\infty}^{\tau} I\left(0, \tau^{\prime}\right) d \tau^{\prime}\right. \\
& \left.+\int_{0}^{\zeta} h \nu_{l} \int_{-\infty}^{\tau} S\left(\zeta^{\prime}, \tau^{\prime}\right) d \tau^{\prime} d \zeta^{\prime}\right) \\
& \times\left[s \int _ { - \infty } ^ { \tau } I ( 0 \tau ^ { \prime } ) \operatorname { e x p } \left(s \int_{-\infty}^{\tau^{\prime}} I\left(0, \tau^{\prime \prime}\right) d \tau^{\prime \prime}\right.\right. \\
& \left.\left.+\int_{0}^{\zeta} h \nu_{l} s \int_{-\infty}^{\tau^{\prime}} S\left(\zeta^{\prime}, \tau^{\prime \prime}\right) d \tau^{\prime \prime} d \zeta^{\prime}\right) d \tau^{\prime}+1\right]^{-1} .
\end{aligned}
$$

This is our principal result. It is an explicit expression for the output intensity in a laser amplifier having an arbitrary input intensity $I(0, \tau)$ and an arbitrary spaceand time-dependent pump $S(\zeta, \tau)$. The following examples include both stationary- and traveling-wave pump distributions.

\section{EXPONENTIALLY PUMPED LASERS}

To investigate the implications of Eq. (16), it is necessary to assume a model for the pumping function $S(\zeta, \tau)$. A function which is often useful in the analysis of practical pulsed lasers is one which after initiation decays exponentially in time. This exponential shape can arise when the actual pump source (discharge, electron beam, optical pump, etc.) decays exponentially or when the transfer of excitation from some other level of the system decays exponentially. A general travelingwave exponential pump source can be written in the 
form

$$
\begin{aligned}
S(z, t) & =\frac{g_{0}}{h \nu_{l} s \tau_{3}} \exp \left(-\frac{t}{\tau_{3}}+\frac{z}{v_{e} \tau_{3}}\right), & & t-\frac{z}{v_{e}}>0 \\
& =0, & & t-\frac{z}{v_{e}}<0,
\end{aligned}
$$

where $v_{e}$ is the velocity of the excitation pulse. In the transformed coordinates $\mathrm{Eq}$. (17) is

$$
\begin{aligned}
S(\zeta, \tau) & =\frac{g_{0}}{h \nu_{l} s \tau_{3}} \exp \left(-\frac{\tau}{\tau_{3}}-\frac{\zeta}{v_{g} \tau_{3}}+\frac{\zeta}{v_{e} \tau_{3}}\right), & & \tau+\frac{\zeta}{v_{g}}-\frac{\zeta}{v_{e}}>0 \\
& =0, & & \tau+\frac{\zeta}{v_{g}}-\frac{\zeta}{v_{e}}<0 .
\end{aligned}
$$

Using Eq. (18), the inner integrals in Eq. (16) can be readily performed. The results are tedious to express, however, since the form of the solution depends on whether $\tau$ is greater or less than $\zeta\left(v_{e}^{-1}-v_{g}^{-1}\right)$ and also on whether $v_{e}$ is greater or less than $v_{g}$. In the limit $v_{e}$ $\gg v_{g}$ the result is

$$
\begin{aligned}
I(\zeta, \tau)= & I(0, \tau) \exp \left\{s \int_{0}^{\tau} I\left(0, \tau^{\prime}\right) d \tau^{\prime}+g_{0} v_{g} \tau_{3}\left[\exp \left(-\frac{\tau}{\tau_{3}}-\frac{\zeta}{\tau_{2} v_{g}}\right)\right.\right. \\
& \left.\left.-\exp \left(-\frac{\tau}{\tau_{3}}\right)\right]\right\}\left(s \int _ { 0 } ^ { \tau } I ( 0 , \tau ) \operatorname { e x p } \left\{s \int_{0}^{\tau^{\prime}} I\left(0, \tau^{\prime \prime}\right) d \tau^{\prime \prime}\right.\right. \\
& \left.+g_{0} v_{g} \tau_{3}\left[\exp \left(-\frac{\tau^{\prime}}{\tau_{3}}-\frac{\zeta}{\tau_{2} v_{g}}\right)-\exp \left(-\frac{\tau^{\prime}}{\tau_{3}}\right)\right]\right\} d \tau^{\prime} \\
& \left.+\exp \left(-g_{0} \zeta\right)\right)^{-1}
\end{aligned}
$$

provided that the intensity is zero prior to $\tau=0$. This limit corresponds to the case of uniform pumping over the entire length of the amplifier.

Plots of Eq. (19) are given in Fig. 1 where the pulse shape is shown as a function of the time coordinate $\tau$ for various amplifier lengths $\zeta$. The input-pulse shape used in these plots is

$$
\begin{aligned}
I(0, \tau) & =I_{1}, & & \tau>0 \\
& =0, & & \tau<0 .
\end{aligned}
$$

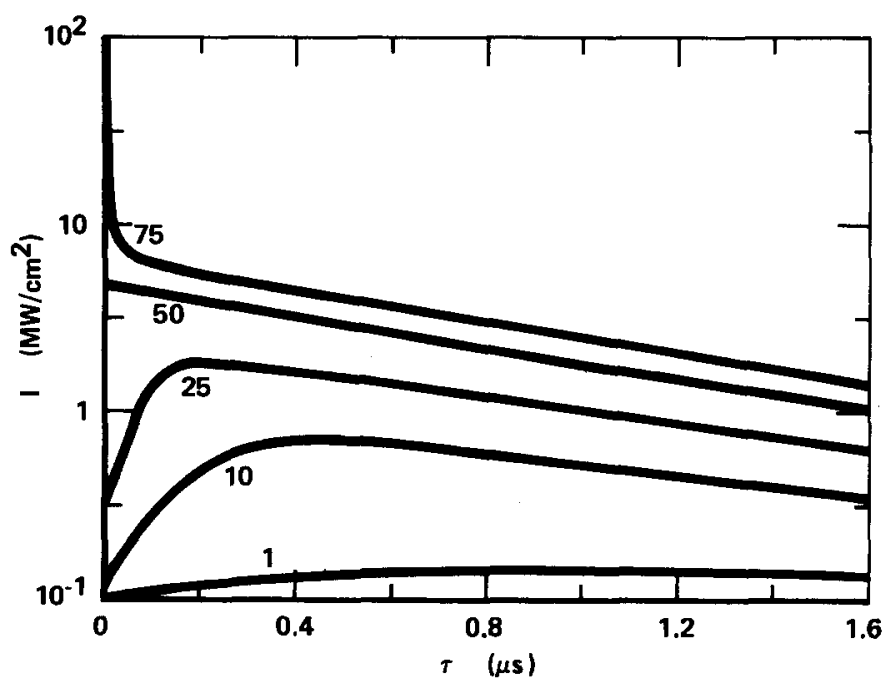

FIG. 1. Output intensity as a function of time for various amplifier lengths in a laser with a uniform exponentially decaying pump rate and a step-function input.

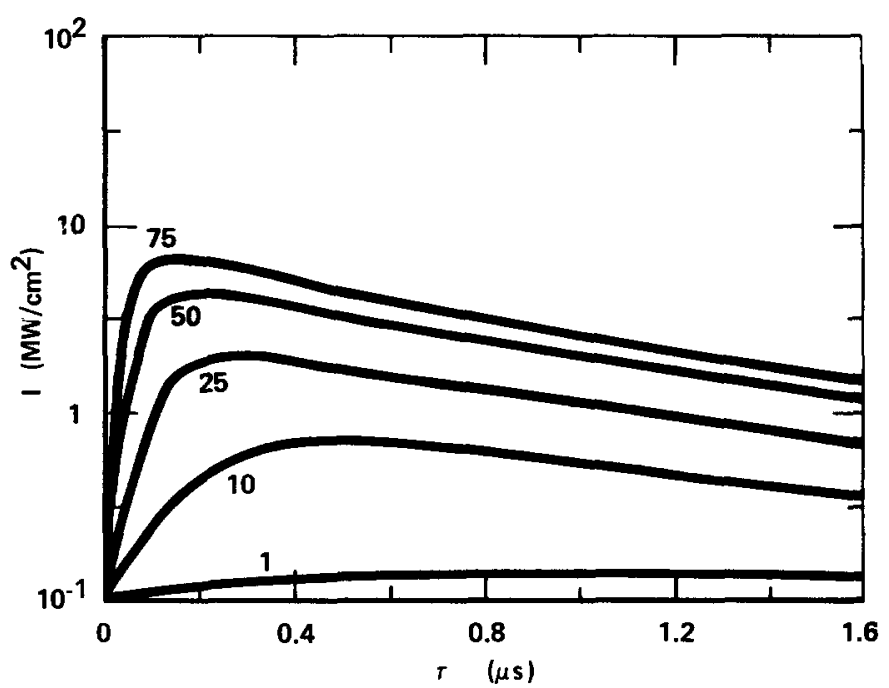

FIG. 2. Output intensity as a function of time for various amplifier lengths in a laser with a traveling-wave exponentially decaying pump rate and a step-function input.

This step function is the simplest shape to study analy$t$ ically, and it is sufficient to illustrate most of the essential features of the amplifier transfer characteristics. With Eq. (20), Eq. (19) reduces to

$$
\begin{aligned}
I(\zeta, \tau)= & I_{1} \exp \left\{s I_{1} \tau+g_{0} v_{g} \tau_{3}\left[\exp \left(-\frac{\tau}{\tau_{3}}-\frac{\zeta}{\tau_{2} v_{g}}\right)-\exp \left(-\frac{\tau}{\tau_{3}}\right)\right]\right\} \\
& \times\left(s I _ { 1 } \int _ { 0 } ^ { \tau } \operatorname { e x p } \left\{s I_{1} \tau^{\prime}+g_{0} v_{g} \tau_{3}\left[\exp \left(-\frac{\tau^{\prime}}{\tau_{3}}-\frac{\zeta}{\tau_{2} v_{g}}\right)\right.\right.\right. \\
& \left.\left.-\exp \left(-\frac{\tau^{\prime}}{\tau_{3}}\right)\right\} d \tau^{\prime}+\exp \left(-g_{0} \zeta\right)\right)^{-1}
\end{aligned}
$$

The numerical constants used in constructing Fig. 1 a re approximately appropriate for TEA $\mathrm{CO}_{2}$ lasers (within the limitations of the two-level model of the laser transition). In particular we have used the values $s=10^{-3} \mathrm{~m}^{2} / \mathrm{J}, I_{1}=10^{9} \mathrm{~W} / \mathrm{m}^{2}, g_{0}=1 \mathrm{~m}^{-1}, v_{g}=3 \times 10^{8} \mathrm{~m} / \mathrm{s}$, $\tau_{3}=1 \mu \mathrm{s}$, and the length $\zeta$ is measured in $\mathrm{m}$. Several features of the plots are significant. For short distances the leading edge of the pulse $(\tau=0)$ experiences no gain, while the decay of the later portion of the pulse shows that the inversion eventually becomes depleted. With greater amplification lengths the leading edge does experience gain, and the pulse maximum moves forward. This change of behavior results because the remote portions of the amplifier receive substantial pumping before the arrival of the light pulse. For distances greater than about $50 \mathrm{~m}$ a "shock front" develops with the energy maximum at $\tau=0$.

A second limit of practical interest occurs when the pump pulse propagates along the laser amplifier at the same velocity as the optical signal. With short-lived laser states or self-termination (fast filling of the lower level), this is the only way to obtain efficient pulse amplification. In the limit $v_{e}=v_{g}$ the exponential pump pulse of $\mathrm{Eq}$. (18) reduces to

$$
\begin{aligned}
S(\zeta, \tau) & =\frac{g_{0}}{h \nu_{l} s \tau_{3}} \exp \left(-\frac{\tau}{\tau_{3}}\right), & & \tau>0 \\
& =0, & & \tau<0
\end{aligned}
$$


Therefore, the intensity of Eq. (16) can be written

$$
\begin{aligned}
I(\zeta, \tau)= & I(0, \tau) \exp \left\{s \int_{0}^{\tau} I\left(0, \tau^{\prime}\right) d \tau^{\prime}+g_{0} \zeta\left[1-\exp \left(-\frac{\tau}{\tau_{3}}\right)\right]\right\} \\
& \times\left(s \int _ { 0 } ^ { \tau } I ( 0 , \tau ^ { \prime } ) \operatorname { e x p } \left\{s \int_{0}^{\tau^{\prime}} I\left(0, \tau^{\prime \prime}\right) d \tau^{\prime \prime}\right.\right. \\
& \left.\left.+g_{0} \xi\left[1-\exp \left(-\frac{\tau^{\prime}}{\tau_{3}}\right)\right]\right\} d \tau^{\prime}+1\right)^{-1}
\end{aligned}
$$

where again it is assumed that the intensity is zero prior to $\tau=0$. With the step-function input pulse of $\mathrm{Eq}$ (20) this is

$$
\begin{aligned}
I(\zeta, \tau)= & I_{1} \exp \left\{s I_{1} \tau+g_{0} \zeta\left[1-\exp \left(-\frac{\tau}{\tau_{3}}\right)\right]\right\} \\
& \times\left(s I_{1} \int_{0}^{\tau} \exp \left\{s I_{1} \tau^{\prime}+g_{0} \zeta\left[1-\exp \left(-\frac{\tau^{\prime}}{\tau_{3}}\right)\right]\right\} d \tau^{\prime}+1\right)^{-1} .
\end{aligned}
$$

Plots of Eq. (24) are given in Fig. 2 using the same numerical values as in the previous example. The initial behavior of the transmitted pulse is the same as in the case described previously. With increasing values of the amplifier length $\zeta$, the intensity maximum advances toward $\tau=0$. No shock front develops now, however, because the leading edge of the pulse always experiences zero gain. It is shown in Sec. VI that $\mathrm{Eq}$. (24) (or other traveling-wave solutions) also represent the behavior of mirrorless traveling-wave lasers in which the input is spontaneous-emission noise. It is only necessary to replace the input $I_{1}$ by the noise parameter $I_{0}=\pi h \nu_{l} \Delta v_{h} / 2 A$. These results provide the most complete analytic solutions for several recent lasers including the ultraviolet (uv) Blumlein pumped $\mathrm{N}_{2}$ and $\mathrm{H}_{2}$ lasers $^{5,6}$ as well as longitudinal electron-beampumped devices. ${ }^{7}$

\section{PREPUMPED LASERS}

With long-lived laser levels if becomes possible to complete the pumping of the laser transition prior to introduction of the optical pulse. This technique provides the greatest pulse efficiency and the maximum amount of pulse sharpening. To explore this limit in detail it is assumed that the laser is excited by the exponential pulse

$$
\begin{aligned}
S(z, t) & =\frac{g_{0}(z)}{h \nu_{l} s \tau_{3}} \exp \left(-\frac{t}{\tau_{3}}+\frac{t_{p}}{\tau_{3}}\right), & & t>t_{p} \\
& =0, & & t<t_{p},
\end{aligned}
$$

where $t_{p}$ is the initiation time for the pump pulse. In the transformed coordinates $\mathrm{Eq} .(25)$ is

$$
\begin{aligned}
S(z, t) & =\frac{g_{0}(\zeta)}{h \nu_{t} s \tau_{3}} \exp \left(-\frac{\tau}{\tau_{3}}-\frac{\zeta}{v_{g} \tau_{3}}+\frac{t_{p}}{\tau_{3}}\right), & \tau+\frac{\zeta}{v_{g}}>t_{p} \\
& =0, & \tau+\frac{\zeta}{v_{g}}<t_{p} .
\end{aligned}
$$

With Eq. (26), Eq. (16) becomes

$$
\begin{aligned}
I(\zeta, \tau)= & I(0, \tau) \exp \left\{s \int_{0}^{\tau} I\left(0, \tau^{\prime}\right) d \tau^{\prime}\right. \\
& \left.+\int_{0}^{\zeta} g_{0}\left(\zeta^{\prime}\right)\left[1-\exp \left(-\frac{\tau}{\tau_{3}}-\frac{\zeta^{\prime}}{v_{g} \tau_{3}}+\frac{t_{p}}{\tau_{3}}\right)\right] d \zeta\right\}
\end{aligned}
$$

$$
\begin{aligned}
& \times\left(: \int_{0}^{\tau} I\left(0, \tau^{\prime}\right) \exp \left\{s \int_{0}^{\tau^{\prime}} I\left(0, \tau^{\prime \prime}\right) d \tau^{\prime \prime}\right.\right. \\
& \left.\left.+\int_{0}^{\zeta} g_{0}\left(\zeta^{\prime}\right)\left[1-\exp \left(-\frac{\tau^{\prime}}{\tau_{3}}-\frac{\zeta^{\prime}}{r_{g} \tau_{3}}+\frac{l_{p}}{\tau_{3}}\right)\right] d \xi^{\prime}\right\} d \tau^{\prime}+1\right)^{-1}
\end{aligned}
$$

where it has been assumed that the intensity is zero prior to $\tau=0$ and that the pump initiation time $l_{p}$ is negative. If the pump pulse occurs very early $\left(l_{p} \rightarrow-\infty\right)$ this is

$$
\begin{aligned}
I(\zeta, \tau)= & I(0, \tau) \exp \left[s \int_{0}^{\tau} I\left(0, \tau^{\prime}\right) d \tau^{\prime}\right] \\
& \times\left\{s \int_{0}^{\tau} I\left(0, \tau^{\prime}\right) \exp \left[s \int_{0}^{\tau} I\left(0, \tau^{\prime \prime}\right) d \tau^{\prime \prime}\right]\right. \\
& \left.+\exp \left[-\int_{0}^{\zeta} g_{0}\left(\zeta^{\prime}\right) d \zeta^{\prime}\right]\right\}^{-1},
\end{aligned}
$$

where the numerator and denominator have been multiplied by the factor $\exp \left[-\int_{0}^{\xi} g_{0}\left(\zeta^{\prime}\right) d \xi^{\prime}\right]$. The denominator can be integrated by parts yielding

$$
\begin{aligned}
I(\zeta, \tau)= & I(0, \tau) \exp \left[s \int_{0}^{\tau} I\left(0, \tau^{\prime}\right) d \tau^{\prime}\right] \\
& \times\left\{\exp \left\lfloor s \int_{0}^{\tau} I\left(0, \tau^{\prime}\right) d \tau^{\prime}\right]+\exp \left[-\int_{0}^{\xi} g_{0}\left(\zeta^{\prime}\right) d \zeta^{\prime}\right]-1\right\}^{(-1}
\end{aligned}
$$

and this special case of our propagation formulas is equivalent to equations which have been given previously. ${ }^{1-3}$

Equation (29) is illustrated first for the special case of a step-function input pulse. More realistic laseroscillator pulses are then considered. With the step pulse of $\mathrm{Eq},(20)$, and a $\zeta$-independent gain $g_{0}, \mathrm{Eq}$ (29) reduces to

$$
I(\zeta, \tau)=\frac{I_{1} \exp \left(s I_{1} \tau\right)}{\exp \left(s I_{1} \tau\right)+\exp \left(-g_{0} \zeta\right)-1}
$$

Equation (30) is plotted in Fig. 3 using the same values as employed previously. Even for short amplifiers the leading edge of the pulse experiences the greatest gain and depletes the population inversion seen by the remainder of the pulse.

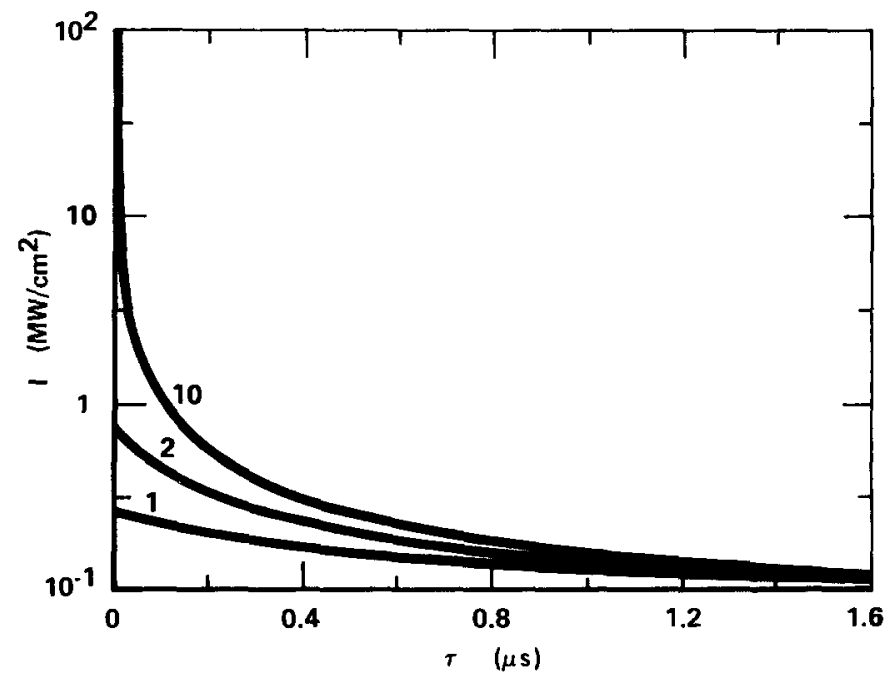

FIG. 3. Output intensity as a function of time for various amplifier lengths in a prepumped laser with a step-function input. 
For a more realistic example we consider now the case that the input pulse is generated by a low-loss laser oscillator with instantaneous gain switching. Thus the input intensity is governed by ${ }^{4}$

$$
\begin{aligned}
I(0, \tau) & =\frac{0.2 I_{1}\left(g_{c}+2 s I_{0}\right)}{g_{c} \exp \left[-\left(g_{c}+2 s I_{0}\right) \tau\right]+2 s I_{0}}, & & \tau>0 \\
& =0, & & \tau<0,
\end{aligned}
$$

where $20 \%$ coupling has been assumed. Equation (29) is plotted in Fig. 4 using $\mathrm{Eq}$. (31) and the previous numerical values $s=10^{-3} \mathrm{~m}^{2} / \mathrm{J}, I_{0}=10^{-6} \mathrm{~W} / \mathrm{m}^{2}, g_{0}=1 \mathrm{~m}^{-1}$, and $g_{c}=1.5 \times 10^{8} \mathrm{~s}^{-1}$. It is apparent from the figure that the primary effect of the amplifier is to enhance the leading edge of the pulse without affecting the trailing edge. Thus the pulse appears to advance ahead of the position it would have if propagating at the velocity $v_{g}$. One readily finds that the peak value of the pulses shown in the figure is $I_{\text {peak }}=g_{c} / s=1.5 \times 10^{11} \mathrm{~W} / \mathrm{m}^{2}$. Since we have assumed that the pulse begins abruptly at $\tau=0$, a shock front ultimately forms at this time indicating that spontaneous emission in the amplifier itself has also ceased to be negligible.

$\mathrm{T}$ ie similar shape of the curves in Fig. 4 suggests that a steady-state-pulse solution should be possible propagating at a velocity $v_{s}$ greater than the velocity $v_{s}$, and this solution can be readily obtained. If the intensity, population inversion and pump are functions of only the new variable $T=t-z / v_{3}$, Eqs. (1) and (2) can be written

$$
\begin{aligned}
& \left(\frac{1}{v_{s}}-\frac{1}{v_{s}}\right) \frac{d I(T)}{d T}=h v_{s} s I(T) N(T), \\
& \frac{d N(T)}{d T}=S(T)-s I(T) N(T),
\end{aligned}
$$

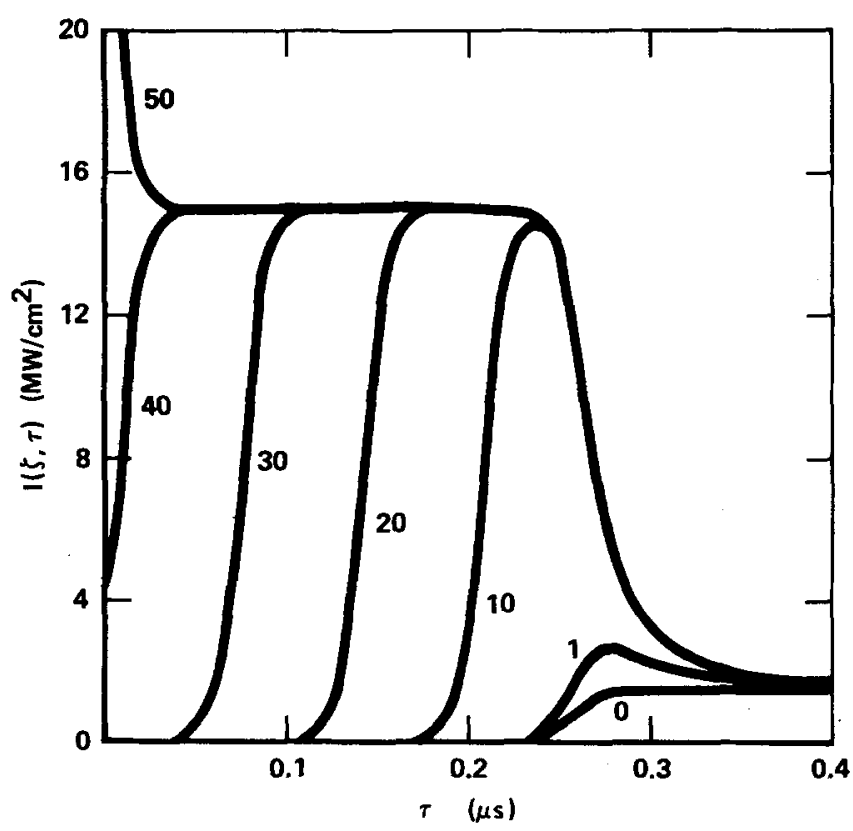

FIG. 4. Output intensity as a function of time for various amplifier lengths in a prepumped laser with an input pulse from an instantaneously gain-switched laser oscillator. where loss and spontaneous relaxation terms have been set equal to zero. These equations can be solved directly by techniques similar to those employed in Sec. II. However, in a prepumped laser we have $S(T)=0$, and Eqs. (32) and (33) may be combined to obtain

$$
\frac{d I(T)}{d T}=g_{c} I(T) \exp \left(-s \int_{-\infty}^{T} I\left(T^{\prime}\right) d T^{\prime}\right)
$$

where the gain coefficient

$$
g_{c}=\frac{h \nu_{1} s N_{0}}{v_{g}^{-i}-v_{s}^{-1}}
$$

has been introduced.

The integral of $\mathrm{Eq} .(35)$ is

$$
s I(T)=-g_{c} \exp \left[-s \int_{-\infty}^{T} I\left(T^{\prime}\right) d T^{\prime}\right]+g_{c}
$$

and the boundary condition $I(-\infty)=0$ has been imposed In terms of the definition $C(T)=s \int_{-\infty}^{T} I\left(T^{\prime}\right) d T^{\prime}$ this can be written

$$
\frac{d C}{1-\exp (-C)}=g_{c} d T
$$

and the integral of this equation is

$$
C(T)=\ln \left[1+\alpha \exp \left(g_{c} T\right)\right]
$$

where $\alpha$ is an integration constant. Therefore the intensity distribution of the stationary-pulse solution is

$$
s I(T)=\frac{\alpha g_{c} \exp \left(g_{c} T\right)}{1+\alpha \exp \left(g_{c} T\right)} .
$$

With an initial condition at $T=0, E q .(39)$ is

$$
I(T)=\frac{I(0) g_{c} \exp \left(g_{c} T\right)}{g_{c}-s I(0)+s I(0) \exp \left(g_{c} T\right)} .
$$

This is a rounded step function of the type implied by Fig. 4, and as expected the maximum value is $g_{c} / s$.

It follows from $\mathrm{Eq}$. (35) that the apparent velocity of the stationary-pulse solution is directly related to the exponential growth constant of the leading edge $g_{c}$ according to

$$
\frac{v_{s}}{v_{s}}=\left(1-\frac{v_{s} g_{0}}{g_{c}}\right)^{-1} \text {. }
$$

This result differs significantly from a previously given expression for the effective pulse velocity in a saturating amplifier ${ }^{10}$

$$
\frac{v_{s}}{v_{s}}=1+\frac{v_{c} g_{0}}{g_{c}}
$$

In particular Eq. (41) shows that the velocity of an exponential pulse may be infinite or negative, while the velocity in $\mathrm{Eq} .(42)$ is always positive. A negative velocity simply implies that with sufficiently high gain a particular intensity level on the exponential leading front emerges from the amplifier before the same intensity level enters the amplifier. This situation is illustrated in Fig. 5 using numbers appropriate to our $\mathrm{CO}_{2}$ model $\left(v_{\mathrm{s}}=3 \times 10^{8} \mathrm{~m} / \mathrm{s}, g_{0}=1 \mathrm{~m}^{-1}\right.$, and $\left.g_{c}=1.5 \times 10^{8} \mathrm{~s}^{-1}\right)$. It follows from Eqs. (40) and (41) that the spatial depen- 


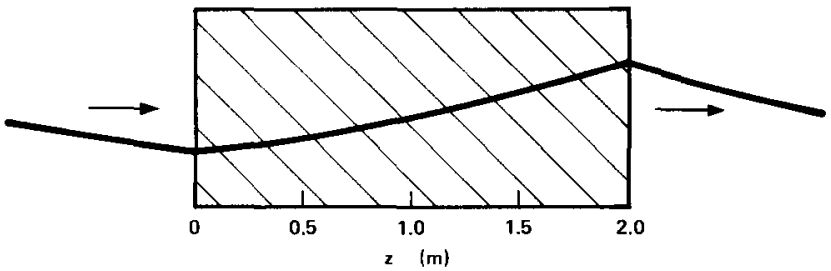

FIG. 5. Instantaneous intensity of an exponentially growing pulse in an unsaturated laser amplifier. The fact that a particular intensity level emerges from the amplifier before that same intensity enters the amplifier implies that the effective "velocity" with in the amplifier is negative.

dence of the intensity inside of the amplifier prior to saturation is governed by $\exp \left(g_{0}-g_{c} / v_{s}\right) z=\exp (0.5 z)$, while the intensity outside varies like $\exp \left(-g_{c} z / v_{g}\right)$ $=\exp (-0.5 z)$. A negative propagation velocity is similar to the behavior of laser amplifiers in spectral regions of anomalous dispersion where the group velocity itself may be negative. ${ }^{11}$ In fact the familiar group velocity formula

$$
\frac{v_{g}}{v_{\mathrm{ph}}}=\left(1+\frac{\nu}{n} \frac{d n}{d \nu}\right)^{-1}
$$

is in the same form as Eq. (41), and ordinarily the dispersion correction $d n / d \nu$ is also proportional to the gain $g_{0}$ 。

The input-pulse shape assumed in Fig. 4 has an exponential leading edge, and this case corresponds to the output pulse from a laser oscillator with instantaneous switching of the gain or loss. In some lasers the gain does not normally switch on instantaneously, and this situation is illustrated in Fig. 6. Here we have used the input-pulse shape ${ }^{4}$

$$
\begin{aligned}
I(0, \tau) & =0.2 I_{0} \exp \left\{g_{c} \tau-g_{c} \tau_{3}\left[1-\exp \left(-\frac{\tau}{\tau_{3}}\right)\right]+2 s I_{0} \tau\right\} \\
& \times\left(2 s I _ { 0 } \int _ { 0 } ^ { \tau } \operatorname { e x p } \left\{g_{c} \tau^{\prime}-g_{c} \tau_{3}\left[1-\exp \left(-\frac{\tau^{\prime}}{\tau_{3}}\right)\right]\right.\right. \\
& \left.\left.+2 s I_{0} \tau^{\prime}\right\} d \tau^{\prime}+1\right)^{-1}, \quad \begin{array}{l}
\tau>0, \\
=0,
\end{array}
\end{aligned}
$$

in $\mathrm{Eq}$. (29) together with the numerical values $g_{c}$ $=1.5 \times 10^{8} \mathrm{~s}^{-1}, \tau_{3}=10^{-6} \mathrm{~s}, s=10^{-3} \mathrm{~m}^{2} / \mathrm{J}$, and $I_{0}=10^{-6} \mathrm{~W} /$ $\mathrm{m}^{2}$. The constant $\tau_{3}$ is the exponential growth constant of the unsaturated gain, and the factor 0.2 represents the output coupling of the laser oscillator. There are some similarities between Figs. 4 and 6 , but there are also some obvious differences. As the leading front advances in the laser amplifier the peak amplitude diminishes because the slope of the leading edge is reduced. If the slope were greater earlier in the pulse, the opposite limit of pulse sharpening would occur. Since abrupt switching has been assumed at $\tau=0$, a shock eventually forms at this time.

\section{PULSE SHAPING}

For some applications of high-powered laser pulses, precise pulse tailoring is required. A currently fashionable example involves the high-energy pulses of pro- posed laser-fusion reactors. For the pellet-compression devices, numerical simulations suggest that the optimum pulse shape has a time dependence of the form ${ }^{12}$

$$
\begin{aligned}
I(t) & =I_{2}\left(1-t / \tau_{0}\right)^{-2}, & & \tau>0 \\
& =0, & & \tau<0,
\end{aligned}
$$

where $\tau_{0}$ is the pellet collapse time. This type of pulse produces the most efficient shock compression and heating of a spherical DT pellet. There are various ways that such a pulse shape can be achieved. With continuously variable optical shutters it is obvious that any arbitrary pulse shape should be possible. However, a shutter technique is optically inefficient and does not guarantee that additional distortion will not occur in the succeeding laser amplifier stages. In a more practical approach the desired output-pulse shape is synthesized as a superposition of smaller rectangular puls es ${ }^{12}$ The purpose of the present discussion is to show that an arbitrary pulse shape can also be obtained by a proper choice of the space- and time-dependent pumping in the final stages of laser amplification.

Equation (16) is a general relationship expressing the output intensity in terms of the input intensity and the pump distribution $S(\zeta, \tau)$. This formula $c$ an be readily inverted to yield the pump distribution needed to obtain a particular output intensity. To be specific we make the reasonable assumption that the total pump energy density deposited in the amplifier is independent of position. Then if the pump is in the form of a traveling wave $S(\tau)$ and the length of the amplifier is $\zeta_{0}$, Eq. (16) reduces to

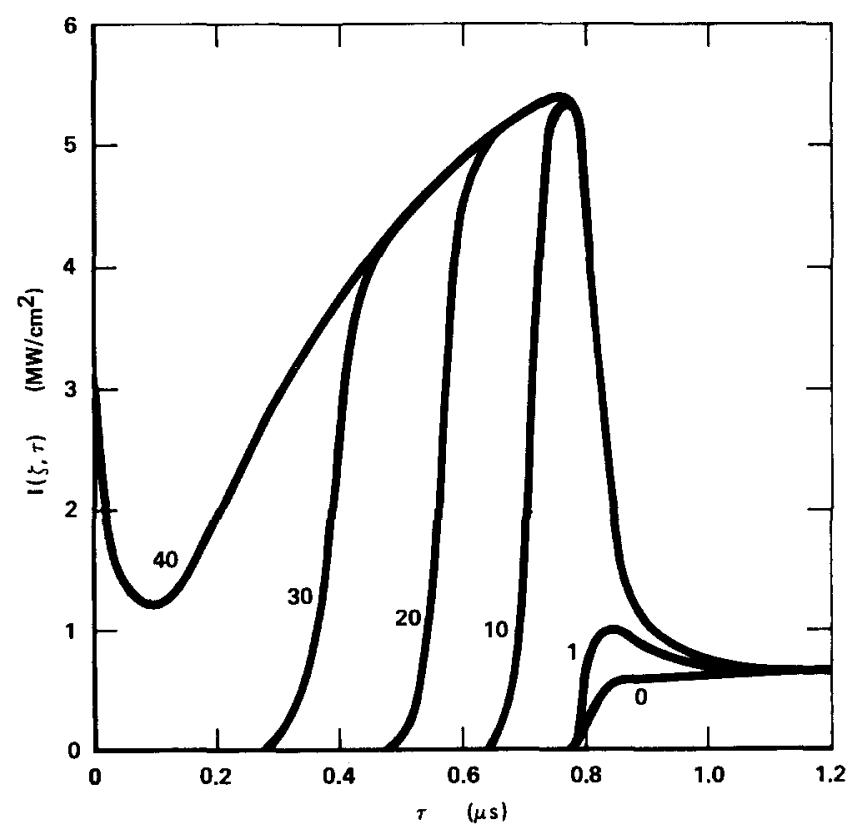

FIG. 6. Output intensity as a function of time for various prepumped amplifer lengths. The input pulse is from a laser oscillator with a gain switching time of $\tau_{3}=1 \mu \mathrm{s}$. 


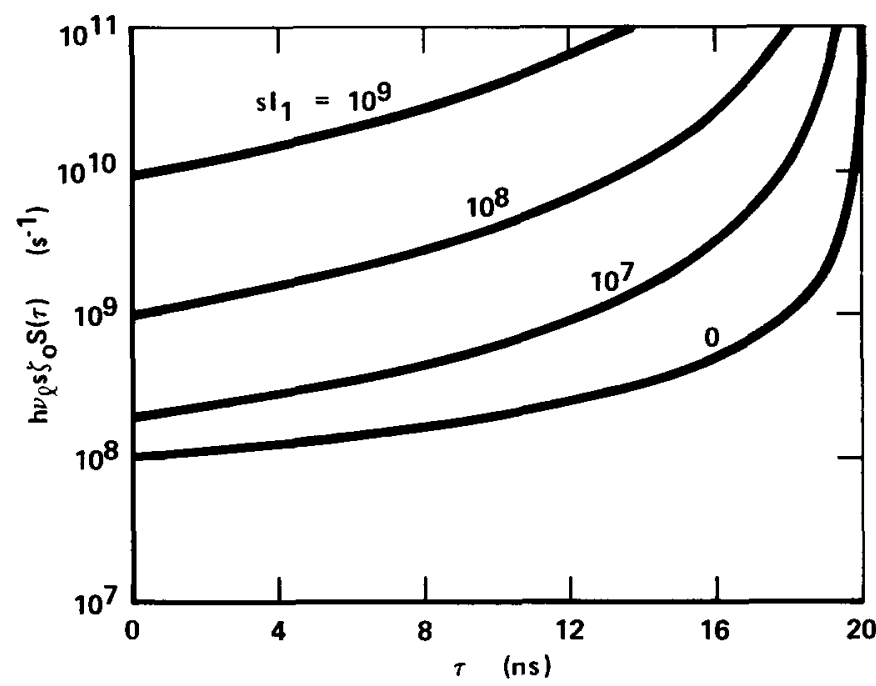

FIG. 7. Pump pulse needed to obtain an "ideal" output-pulse shape for laser fusion.

$$
\begin{aligned}
I\left(\zeta_{0}, \tau\right)= & I(0, \tau) \exp \left[s \int_{-\infty}^{\tau} I\left(0, \tau^{\prime}\right) d \tau^{\prime}+h \nu_{l} s \zeta_{0} \int_{-\infty}^{\tau} S\left(\tau^{\prime}\right) d \tau^{\prime}\right] \\
& \times\left\{s \int _ { - \infty } ^ { \tau } I ( 0 , \tau ^ { \prime } ) \operatorname { e x p } \left[s \int_{-\infty}^{\tau^{\prime}} I\left(0, \tau^{\prime \prime}\right) d \tau^{\prime \prime}\right.\right. \\
& \left.\left.+h \nu_{l} s \zeta_{0} \int_{-\infty}^{\tau^{\prime}} S\left(\tau^{\prime \prime}\right) d \tau^{\prime \prime}\right] d \tau^{\prime}+1\right\}^{-1} \\
= & \frac{1}{s} \frac{\partial}{\partial \tau} \ln \left\{s \int _ { - \infty } ^ { \tau } I ( 0 , \tau ^ { \prime } ) \operatorname { e x p } \left[s \int_{-\infty}^{\tau^{\prime}} I\left(0, \tau^{\prime \prime}\right) d \tau^{\prime \prime}\right.\right. \\
& \left.\left.+h \nu_{l} s \zeta_{0} \int_{-\infty}^{\tau^{\prime}} S\left(\tau^{\prime \prime}\right) d \tau^{\prime \prime}\right] d \tau^{\prime}+1\right\}
\end{aligned}
$$

An integration and exponentiation yield

$$
\begin{aligned}
& s \int_{-\infty}^{\tau} I\left(0, \tau^{\prime}\right) \exp \left[s \int_{-\infty}^{\tau^{\prime}} I\left(0, \tau^{\prime \prime}\right) d \tau^{\prime \prime}\right. \\
& \left.\quad+h \nu_{l} s \zeta_{0} \int_{-\infty}^{\tau^{\prime}} S\left(\tau^{\prime \prime}\right) d \tau^{\prime \prime}\right] d \tau^{\prime}+1=\exp \left[s \int_{-\infty}^{\tau} I\left(\zeta_{0}, \tau^{\prime}\right) d \tau^{\prime}\right] .
\end{aligned}
$$

Differentiation with respect to $\tau$ gives

$$
\begin{aligned}
& s I(0, \tau) \exp \left[s \int_{-\infty}^{\tau} I\left(0, \tau^{\prime}\right) d \tau^{\prime}+h \nu_{l} s \zeta_{0} \int_{-\infty}^{\tau} S\left(\tau^{\prime}\right) d \tau^{\prime}\right] \\
& =s I\left(\zeta_{0}, \tau\right) \exp \left[s \int_{-\infty}^{\tau} I\left(\zeta_{0}, \tau^{\prime}\right) d \tau^{\prime}\right]
\end{aligned}
$$

or

$$
\begin{aligned}
& \exp \left(h \nu_{l} s \zeta_{0} \int_{-\infty}^{\tau} S\left(\tau^{\prime}\right) d \tau^{\prime}\right) \\
& \quad=\frac{I\left(\zeta_{0}, \tau\right)}{I(0, \tau)} \exp \left(s \int_{-\infty}^{\tau}\left[I\left(\zeta_{0}, \tau^{\prime}\right)-I\left(0, \tau^{\prime}\right)\right] d \tau^{\prime}\right) .
\end{aligned}
$$

Taking the logarithm of both sides and differentiating again leads to the final result

$S(\tau)=\frac{1}{h \nu_{l} s \zeta_{0}}\left[\frac{\partial}{\partial \tau} \ln \left(\frac{I(\xi, \tau)}{I(0, \tau)}\right)+s\left[I\left(\zeta_{0}, \tau\right)-I(0, \tau)\right]\right]$.

Equation (49) is an explicit expression for the traveling-wave pump distribution needed to obtain an arbitrary output intensity pulse from an arbitrary input intensity pulse. To illustrate the procedure, we consider again the example of pellet-compression laser fusion. The simplest reasonable input pulse is the step function given in $\mathrm{Eq}$ 。(20). Combining Eqs. (20), (45), and $(50)$ one concludes that the required pump function is

$$
\begin{array}{rlrl}
S(\tau)= & \frac{1}{h \nu_{l} s \zeta_{0}}\left\{\frac{\partial}{\partial \tau} \ln \left[\frac{I_{2}}{I_{1}}\left(1-\frac{\tau}{\tau_{0}}\right)^{-2}\right]\right. \\
& \left.+s\left[I_{2}\left(1-\frac{\tau}{\tau_{0}}\right)^{-2}-I_{1}\right]\right\}, & \tau>0 \\
=0, & \tau<0 .
\end{array}
$$

Performing the differentiation yields

$$
\begin{aligned}
S(\tau) & =\frac{1}{h \nu_{l} s \zeta_{0}}\left\{\frac{2}{\tau_{0}-\tau}+s\left[I_{2}\left(1-\frac{\tau}{\tau_{0}}\right)^{-2}-I_{1}\right]\right\}, & & \tau>0 \\
& =0, & & \tau<0 .
\end{aligned}
$$

The general features of Eq. (52) can be shown by a basic numerical example, and plots of the equation are given in Fig. 7 assuming an initial gain of $10\left(I_{2}=10 I_{1}\right)$ and a reasonable pellet collapse time of $\tau_{0}=20 \mathrm{~ns}$. The required pump profile in this case resembles closely the shape of the desired output pulse. If the input pulse were to rise gradually instead of being constant as in this example, then the pump function would not have to rise so steeply.

One may inquire next whether pump functions of this type might be realizable with practical lasers. It is clear that useful modulated amplification is only possible if the pump mechanism can be varied on a time scale comparable to the desired variations in the amplifier output. In flashlamp-pumped neodymium-glass lasers the fluorescence rise time is several hundred $\mu \mathrm{s}$, which would be much too slow for fusion pulse applications. With the electron-beam-pumped $1700-\AA$ xenon laser, on the other hand, the gain can be varied significantly on a time scale of $1 \mathrm{~ns}{ }^{13}$ Other modulation techniques are not well developed for this portion of the uv spectrum, and the traveling-wave gain-modulation method may prove to be useful. The short wavelength of the xenon-laser transition makes this device especially interesting for late portions of the pellet-compression fusion process, and modulation of the electronbeam pumping rate is feasible. For slower and more traditional laser applications, gain modulation might be a simple and effective alternative to conventional modulation methods.

\section{UNSATURATED AMPLIFIERS}

The principal complicating factor in the previous analysis of pulse propagation has been the highly nonlinear saturation behavior. In this section the pulse propagation problem is reexamined briefly with saturation effects omitted. Some pulse amplifiers are designed to operate in an unsaturated mode, and this approximation makes it possible to include other effects such as the spontaneous-emission noise input. In long laser amplifiers noise can be a significant factor in the 
output, and with certain traveling-wave devices such as the $\mathrm{N}_{2}$ or $\mathrm{H}_{2}$ molecular lasers noise is the only input.

If the intensity is small, the terms $s \int_{-\infty}^{\tau} I(\zeta, \tau) d \tau^{\prime}$ can be set equal to zero in Eq. (7) yielding the simpler equation

$$
\begin{aligned}
\frac{\partial I(\zeta, \tau)}{\partial \zeta}= & h \nu_{l} s\left[I(\zeta, \tau)+I_{0}\right] \exp \left(-\frac{\tau}{\tau_{2}}\right) \int_{-\infty}^{r} S\left(\zeta, \tau^{\prime}\right) \\
& \times \exp \left(\frac{\tau^{\prime}}{\tau_{2}}\right) d \tau^{\prime}-\gamma I(\zeta, \tau)
\end{aligned}
$$

This may be regarded as a first-order linear differential equation and the general solution is ${ }^{9}$

$$
\begin{aligned}
I(\zeta, \tau)= & \exp \left[-\gamma \zeta+h \nu_{l} s \exp \left(-\frac{\tau}{\tau_{2}}\right)\right. \\
& \left.\times \int_{0}^{\zeta} \int_{-\infty}^{\tau} S\left(\zeta^{\prime}, \tau^{\prime}\right) \times \exp \left(\frac{\tau^{\prime}}{\tau_{2}}\right) d \tau^{\prime} d \zeta^{\prime}\right] \\
& \times\left\{\int_{0}^{\zeta} h \nu_{l} s I_{0} \exp \left(-\frac{\tau}{\tau_{2}}\right) \int_{-\infty}^{\tau} S\left(\zeta^{\prime}, \tau^{\prime}\right) \exp \left(\frac{\tau^{\prime}}{\tau_{2}}\right) d \tau^{\prime}\right. \\
& \times \exp \left[\gamma \zeta^{\prime}-h \nu_{l} s \exp \left(-\frac{\tau}{\tau_{2}}\right)\right. \\
& \left.\left.\times \int_{0}^{\rho^{\prime}} \int_{-\infty}^{\tau} S\left(\zeta^{\prime \prime}, \tau^{\prime}\right) \exp \left(\frac{\tau^{\prime}}{\tau_{2}}\right) d \tau^{\prime} d \zeta^{\prime}\right] d \zeta^{\prime}+I(0, \tau)\right\}
\end{aligned}
$$

For simplicity we assume that the medium is lossless $(\gamma=0)$. Then Eq. (54) may be integrated by parts yielding

$$
\begin{aligned}
I(\zeta, \tau)= & {\left[I(0, \tau)+I_{0}\right] \exp \left[h \nu_{t} s \exp \left(-\frac{\tau}{\tau_{2}}\right)\right.} \\
& \left.\times \int_{0}^{\zeta} \int_{-\infty}^{\tau} S\left(\zeta^{\prime}, \tau^{\prime}\right) \exp \left(\frac{\tau^{\prime}}{\tau_{2}}\right) d \tau^{\prime} d \zeta^{\prime}\right]-I_{0} .
\end{aligned}
$$

When spontaneous emission in the only input, Eq. (55) reduces to

$$
\begin{aligned}
I(\zeta, \tau)= & I_{0}\left\{\operatorname { e x p } \left[h \nu_{l} s \exp \left(-\frac{\tau}{\tau_{2}}\right) \int_{0}^{\zeta} \int_{-\infty}^{\tau} S\left(\zeta^{\prime}, \tau^{\prime}\right)\right.\right. \\
& \left.\left.\times \exp \left(\frac{\tau^{\prime}}{\tau_{2}}\right) d \tau^{\prime} d \zeta^{\prime}\right]-1\right\} .
\end{aligned}
$$

A specific traveling-wave pump function is given in Eq. (18). If this expression with $v_{e}=\infty$ is combined with $\mathrm{Eq}$. (56), one obtains the explicit formula

$$
\begin{array}{rlrl}
\frac{I(\zeta, \tau)}{I_{0}}= & \exp \left(\frac { g _ { 0 } } { 1 - \tau _ { 3 } / \tau _ { 2 } } \left\{\tau_{3} v_{g} \exp \left(-\frac{\tau}{\tau_{3}}\right)\left[\exp \left(\frac{-\zeta}{\tau_{3} v_{g}}\right)-1\right]\right.\right. \\
& \left.\left.-\tau_{2} v_{g} \exp \left(-\frac{\tau}{\tau_{2}}\right)\left[\exp \left(\frac{-\zeta}{\tau_{2} v_{g}}\right)-1\right]\right\}\right), & \tau>0 \\
= & 0, & \tau<0 .
\end{array}
$$

This limit corresponds to spatially independent pumping along the length of the laser. The more interesting case of a velocity-matched traveling-wave pump occurs with $v_{e}=v_{g}$. Then Eqs. (18) and (56) combine to yield

$$
\begin{aligned}
\frac{I(\zeta, \tau)}{I_{0}} & =\exp \left\{\frac{g_{0} \xi}{1-\tau_{3} / \tau_{2}}\left[\exp \left(-\frac{\tau}{\tau_{2}}\right)-\exp \left(-\frac{\tau}{\tau_{3}}\right)\right]\right\}-1, & & \tau>0 \\
& =0, & & \tau<0 .
\end{aligned}
$$

This limit would be appropriate for Blumlein driven $\mathrm{N}_{2}$ or $\mathrm{H}_{2}$ lasers, for example, ${ }^{5,6}$ Equation (58) implies that the peak of the laser output is delayed by the amount $\tau_{\text {max }}=\ln \left(\tau_{2} / \tau_{3}\right)\left(\tau_{3}^{-1}-\tau_{2}^{-1}\right)^{-1}$.

In the limit that the spontaneous relaxation time is long compared to any other time of interest $\left(\tau_{2} \rightarrow \infty\right) \mathrm{Eq}$ 。 (58) may be combined with $\mathrm{Eq}$, (24) to obtain the output characteristics of a saturating traveling-wave amplifier which has no input other than spontaneous emission. From a comparison of these equations it follows that the output is the same as if there were an ordinary input of the form given in $\mathrm{Eq}$. (20) with $I_{1}=I_{0}$. Thus $\mathrm{Eq}$. (24) and Fig, 4 both apply to this important case of a traveling-wave laser with spontaneous-emission input. The intensity maximum occurs at a time less than the pumping time $\tau_{3}$, and if the relaxation time $\tau_{2}$ is much greater than $\tau_{3}$ the relaxation effects are clearly unimportant.

\section{CONCLUSION}

Several new analytic models have been developed for laser pulse amplifiers and mirrorless laser sources. A basic advantage of these models is the inclusion of an arbitrary space- and time-dependent pump function. It thus becomes possible to predict the behavior of lasers in which the pump pulse overlaps the optical pulse. Specific practical examples include the Blumlein driven uv traveling-wave lasers. On the other hand, the same formulas also make it possible to obtain any desired output-pulse shape by proper choice of the pumping conditions in the laser amplifier. Pellet-compression laser fusion has been considered as an example of this pulse-forming technique.

The discussion and analysis in this work has all been presented within the context of pulse amplification. It should be noted that the same techniques and solutions also apply to saturable laser absorbers. Such attenuators are commonly used as laser $Q$-switching media and as high-speed laser-driven shutters. One can visualize applications where a multilevel material is optically pumped and made to absorb by radiation which couples the lower level of the absorbing transition to the ground state. This configuration would be the analog of the pumped amplifiers which have been considered in detail. Finally we mention that the results often apply also to inhomogeneously broadened media. In such materials the different portions of the propagating spectrum interact with different classes of atoms, so the spectral components may be treated independently. As shown previously, the equations governing each part of the spectrum may be in the same form as the equations governing the total intensity in a homogeneously broadened medium. ${ }^{4}$ Thus all of the present solutions apply, and the intensity can be found by a summation over the various spectral components. 


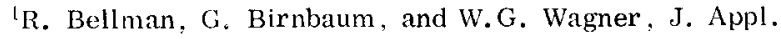
Phys. 34, $780(1963)$.

${ }^{2}$ L. M. Frantz and J.S. Nodvik, J. Appl. Phys. 34, 2346 (196:3)。

E. O. Schulz-DuBois, Bell Syst. Tech.J.43, 625 (1964). ${ }^{4}$ L. W. Casperson, preceding paper, J. Appl. Phys. 47, 4555 (1976)

J. D. Shipman, Appl. Phys. Lett. 10, 3 (1967).

R. W. Waynant, J. D. Shipman, R. C. Elton, and A. W. Ali, Proc. IEEE 59, 679 (1971).

'R.W. Dreyfus and R.T. Hodgson, Phys. Rev. A 9, 2635 (1974) .

${ }^{4}$ L. W. Casperson and A. Yariv, Phys. Rev. Lett. 26, 293 (1971).
${ }^{9}$ W. Kaplan, Ordinary Differential Equations (Addison-Wesley, Palo Alto, Calif., 1962), Eq. (2-58).

${ }^{10}$ N.G. Basov, R.V. Ambartsumyan, V.S. Zuev, P.G. Kryukov, and V.S. Letokhov, Soviet Phys.JETP 23, 16 (1966), Eq. (15).

"See for example, C.G. B. Garrett, and D. E. McCumber, Phys. Rev. A 1, 305 (1970).

${ }^{12} \mathrm{~J}$. Nuckolls, J. Emmett, and L. Wood, Phys. Today 26(No. 8), 46 (1973).

${ }^{13}$ H. A. Koehler, L.J. Ferderber, D. L. Redhead, and P.J. Ebert, Appl。Phys. Lett.21, 198 (1972). 